\title{
Análise zooarqueológica do Sambaqui Lagoa dos Freitas, extremo sul de Santa Catarina, Brasil: primeiros resultados e tendências de exploração de ecossistemas litorâneos durante o Holoceno final
}

\section{Zooarchaeological analysis of the Lagoa dos Freitas Sambaqui, Southern part of Santa Catarina, Brazil: preliminary results and trends in strategies of coastal ecosystems exploitation during the final Holocene}

\author{
DIEGO DIAS PAVEI ${ }^{1,2}$, CAROLINE BORGES $^{1}$, MARCOS CÉSAR PEREIRA SANTOS ${ }^{2}$, \\ JULIANO BITENCOURT CAMPOS ${ }^{2,3} \&$ AMANDA MACHADO DA ROSA $^{2}$
}

'Programa de Pós-graduação em Antropologia (PPGAnt), Universidade Federal de Pelotas (UFPel).

${ }^{2}$ Laboratório de Arqueologia Pedro Ignácio Schmitz (LAPIS)-UNESC, Brasil.

3 Programa de Pós-Graduaçao em Ciências Ambientais (PPGCA)-UNESC.

diego.pavei@unesc.net; arqueocarol@gmail.com;

(Received 31 May 2018; Revised 29 June 2018; Accepted 4 November 2018)

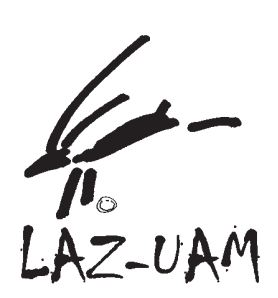

RESUMO: O sítio Lagoa dos Freitas se caracteriza como um concheiro sobre dunas holocênicas e as datas radiocarbônicas obtidas mostram uma cronologia com dois horizontes distintos de ocupação, o primeiro entre 1.360-1.275 cal. AP e o segundo entre 485-305 cal. AP. Com o objetivo de caracterizar a exploração de recursos animais em cada período de ocupação, este trabalho realizou um estudo zooarqueológico dos conjuntos faunísticos vertebrados de cada horizonte, a saber, 4005 restos para a camada I, mais recente, e 10299 restos para a Camada II, mais antiga, somando um total de 14304 restos analisados. Foram identificadas três classes de vertebrados: peixes, mamíferos e aves. Os peixes identificados são em sua maioria bagres marinhos da família Ariidae, robalos da família Centropomidae e corvinas e papa-terras da família Sciaenidae, sendo a corvina (Micropogonias furnieri) a espécie individualmente mais abundante. Para a fauna terrestre, apenas roedores caviomorfos foram identificados, Hidrochoerus hydrochaeris e Ctenomis minutus. Os resultados deste estudo mostram que houve uma exploração mais intensa de ambientes estuarinos em comparação aos ambientes terrestres nas áreas do entorno do sítio e, em especial, o desenvolvimento de atividades de pesca, sem que diferenças significativas tenham sido encontradas entre os diferentes horizontes de ocupação no que se refere a exploração da fauna vertebrada. Estes resultados são importantes porque pouco se conhece das modalidades de apropriação de recursos animais pelos grupos humanos associados aos sambaquis tardios do litoral catarinense.

PALAVRAS CHAVE: ZOOARQUEOLOGIA, SAMBAQUI, PESCA, LITORAL EXTREMO SUL DE SANTA CATARINA

ABSTRACT: The Lagoa dos Freitas site is characterized as a shellmounds on Holocene dunes and the radiocarbon dates obtained show a chronology with two distinct horizons of occupation, the first between 1.360-1.275 cal. AP and the second between 485-305 cal. AP. With the objective of characterizing the exploitation of animal resources in each period of occupation, this work realized out a zooarcheological study of the vertebrate faunal groups of each horizon, namely 4005 
remains for the most recent layer I and 10299 remains for the Layer II , older, adding a total of 14304 analyzed remains. Three classes of vertebrates were identified: fish, mammals and birds. The fish identified are mostly marine catfish of the family Ariidae, snooks and robalos of the family Centropomidae and whitemouth croakers of the family Sciaenidae. The corvina (Micropogonias furnieri) is the most individually abundant specie. For terrestrial fauna, only caviomorphs rodents were identified, Hidrochoerus hydrochaeris and Ctenomis minutus. The results of this study show that there was a more intense exploration of estuarine environments in comparison to terrestrial environments in the areas surrounding the site and, in particular, the development of fishing activities, without significant differences founded between the different horizons of occupation regarding the vertebrate fauna. These results are important because little is known of the modalities of appropriation of animal resources by the human groups associated to the late sambaquis of the Santa Catarina coast

KEYWORDS: ZOOARCHAEOLOGY, SAMBAQUI, FISHING, EXTREME SOUTH COAST OF SANTA CATARINA

\section{INTRODUÇÃO}

O litoral atlântico do estado de Santa Catarina, Brasil, tem sido continuamente povoado desde 7400 anos cal. AP (Giannini et al., 2010). As ocupações humanas pré-coloniais mais frequentes desta região são representadas principalmente pelos sambaquis, sítios arqueológicos construídos a partir do acúmulo antrópico de conchas localizados em áreas de transição ambiental no entorno de estuários, lagunas e lagoas litorâneas (DeBlasis et al., 2007; Giannini et al., 2010). A região do litoral sul de Santa Catarina, representado principalmente pela área da Lagoa de Santa Marta, apresenta uma longa cronologia de ocupação do litoral pelos grupos sambaquieiros entre 7300 e 1500 anos AP. (DeBlasis \& Gaspar, 2009; Giannini et al., 2010). E, nesta região, os sambaquis tem sido o objeto, por mais de 20 anos, de pesquisas focadas no entendimento da complexidade dos grupos sambaquieiros (DeBlasis et al., 1998; Villagrán et al., 2009; Giannini et al., 2010).

No que se refere as análises zooarqueológicas destes sítios, elas demostram que existe uma forte preferência destes grupos pela exploração de ictiofauna marinha-estuarina, embora também sejam explorados mamíferos marinhos e terrestres, aves migratórias, e répteis (Nishida, 2007; Klokler et al., 2010; Cardoso et al., 2014; Cardoso, 2018). Porém pouco se sabe sobre o modo de apropriação de recursos animais em ocupações litorâneas de períodos mais tardios, ao final da ocupação sambaquieira no litoral, entre 2000 e 1000 anos AP, momento também da chegados dos grupos ceramistas a este litoral (DeBlasis et al., 2007). Os únicos estudos zooarqueológicos realizados para este período tardio, o foram para os sítios Içara-01 e Galheta IV, ambos não caracterizados como sambaquis (Rosa, 1999, 2006; Cardoso et al., 2014; Cardoso, 2018). Assim, entender a existência de continuidades ou mudanças nas práticas de apropriação de recursos animais vertebrados para um sítio litorâneo com datações do Holoceno recente na região do litoral extremo sul de Santa Catarina é o objetivo deste trabalho.

\section{CONTEXTO E PROBLEMÁTICA REGIONAL}

O projeto Arqueologia Entre Rios: do Urussanga ao Mampituba (AERUM) tem como foco principal os estudos das ocupações pré-coloniais da região do extremo sul catarinense (Santos et al.; 2016). No que se refere aos estudos zooarquelógicos, nesta área foram estudados conjuntos faunísticos de três sítios, Içara-06, Içara-01 e Sambaqui Lagoa dos Freitas (este estudo). O Içara-06, com 3500 AP, tem a datação mais antiga da área do AERUM e marca o ápice da ocupação sambaquieira na região (Rogge \& Arnt, 2006). O segundo sítio, Içara-01, datado de 1500 anos AP., é considerado um acampamento relacionando ao períodos tardios de ocupação do litoral (Schmitz, 1999). O Içara-06, mais antigo, apresenta um espectro de fauna relacionada com ambientes estuarinos e marinhos com o registro da presença de baleias, lobo-marinhos e pinguins nas amostras, enquanto o Içara-01, mais recente, exibe fauna dulcícola e também fauna estuarina-marinha, além de espécies terrestres pouco comuns para esta região litorânea (Schmitz, 1999; Rosa, 2006; Teixeira, 2006; Tamiozzo et al., 2008). 


\section{O Sambaqui Lagoa dos Freitas}

O sítio arqueológico Sambaqui Lagoa dos Freitas-SLF (SC-ARA-030) se localiza no município de Balneário Rincão, na localidade de Lagoa dos Freitas (UTM 22J 673772/6812135). Situado sobre dunas a 20,5 metros acima do nível do mar, o sambaqui está a uma distância aproximada de 230 metros da Lagoa dos Freitas, 950 metros da Lagoa Urussanga Velha e distante em 1700 metros do mar (Figura 1).

Duas campanhas de escavação foram realizadas. A primeira em 2014 teve como objetivo expor os perfis da área periférica da duna e entender a disposição do sítio sobre a mesma, além da coleta de amostras para datação. Duas áreas foram abertas, a primeira o Setor 1, uma quadra de 4 × 4 m, e a segunda a Trincheira 1 de 10 x $1 \mathrm{~m}$. Em 2015, a segunda campanha de escavação teve o intuito de entender a parte central do sítio e correlacionar com os dados coletados na campanha anterior. Duas trincheiras perpendiculares ( 3 e 4 ) foram abertas no topo do sítio, ambas com $3 \times 1 \mathrm{~m}$.

A estratigrafia e as datações obtidas mostraram que o sambaqui Lagoa dos Freitas se caracteriza pela disposição de duas camadas arqueológicas distintas (Tabela 1) (Santos et al., 2018).

A metodologia utilizada na escavação consistiu em decapagens finas arbitrárias $(2-3 \mathrm{~cm})$ seguindo a declividade do sítio. Todo o sedimento escavado foi peneirado em malhas de 5 e $2 \mathrm{~mm}$ sobrepostas o que permitiu potencializar a recuperação de vestígios faunísticos. Não foram encontradas estruturas de combustão ou funerárias no Sambaqui Lagoa do Freitas e, além da fauna, foram recuperados artefatos líticos $(\mathrm{N}=142)$ associados a uma indústria pouco desenvolvida e realizada, principalmente, em matérias-primas como basalto e arenito (Santos et al., 2018). Na superfície do sítio foram também recuperados fragmentos cerâmicos $(\mathrm{N}=11)$ associados aos grupos Guarani (Santos et al., 2018).

\section{Caracterização ambiental}

A área do entorno do Sambaqui Lagoa dos Freitas apresenta, em gradiente longitudinal, formações vegetais distintas desde restingas até florestas arbóreas de domínio da Mata Atlântica. As áreas mais próximas ao litoral comportam as formações de res-

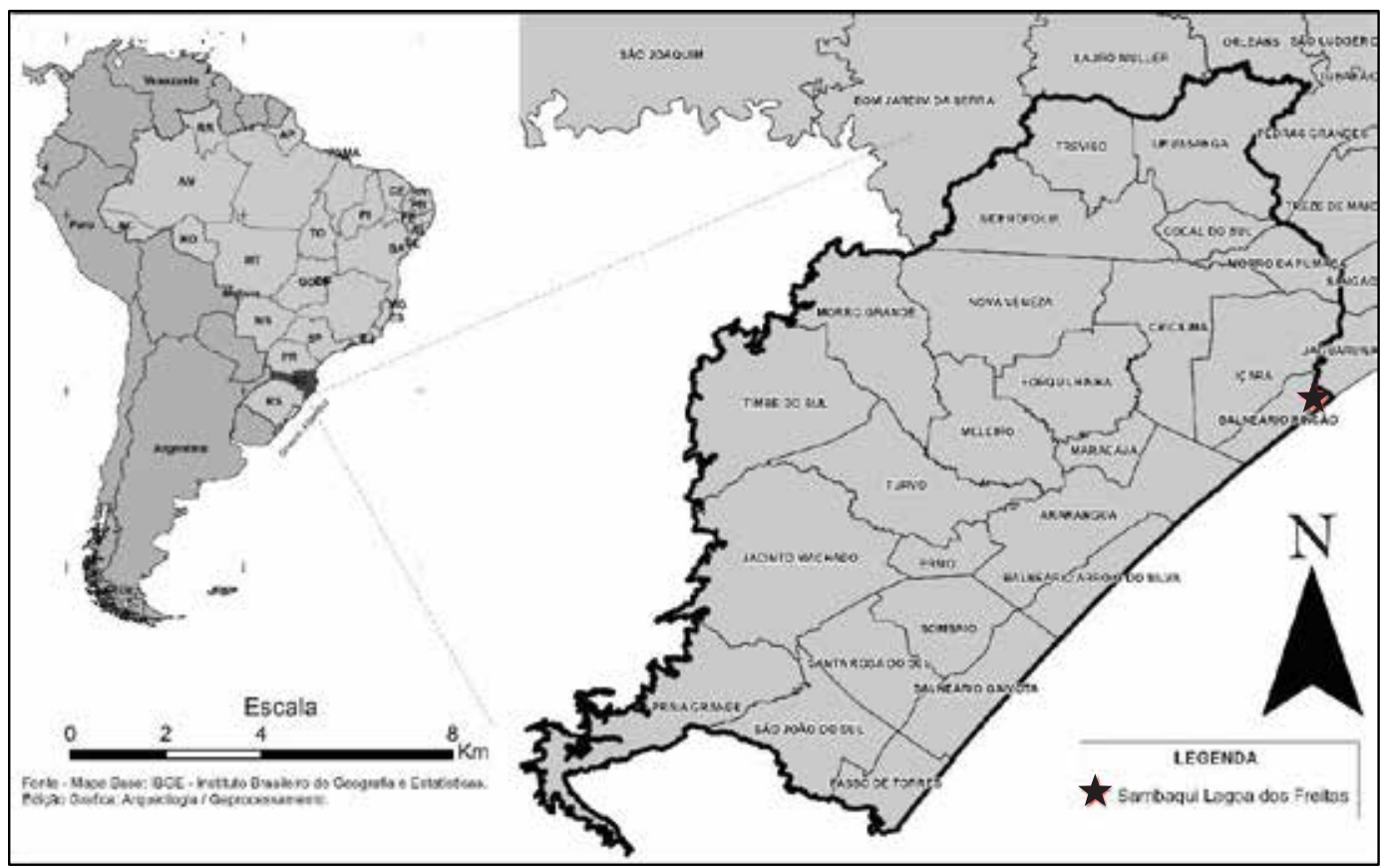

FIGURA 1

Mapa de localização da área de estudo, com o ponto em preto representa a localizaçao do Sambaqui Lagoa dos Freitas (SC-ARA-030). 


\begin{tabular}{|c|c|c|c|c|c|c|}
\hline $\begin{array}{c}\text { Área da } \\
\text { Escavaçao }\end{array}$ & Perfil & Camada & $\begin{array}{c}\text { Data } \\
\text { Convencional }\end{array}$ & $\begin{array}{l}\text { Calibrada } \\
(2 \text { sigmas }) \\
\end{array}$ & $\begin{array}{l}\text { Calibrada } \\
(2 \text { sigmas }) \\
\end{array}$ & Oratórico \\
\hline Trincheira 2 & 5 & 1 & $\begin{array}{c}90+/-30 \mathrm{BP} \\
(95 \% \text { probabilidade) }\end{array}$ & $\begin{array}{l}1930-1880 \\
1865-1845 \\
1835-1810 \\
1720-1710\end{array}$ & $\begin{array}{c}70-20 \\
105-85 \\
140-115 \\
240-230\end{array}$ & $\begin{array}{c}\text { BETA } \\
426852\end{array}$ \\
\hline Trincheira 1 & 1 & 1 & $360+/-30 \mathrm{BP}$ & $1645-1465$ & $485-305$ & $\begin{array}{r}\text { BETA } \\
403221\end{array}$ \\
\hline Trincheira 1 & 1 & 1 & $390+/-30 \mathrm{BP}$ & $1630-1455$ & $495-320$ & $\begin{array}{c}\text { BETA } \\
403220 \\
\end{array}$ \\
\hline Trincheira 3 & 6 & 2 & $1430+/-30 \mathrm{BP}$ & $675-635$ & $1315-1275$ & $\begin{array}{c}\text { BETA } \\
426824 \\
\end{array}$ \\
\hline Setor 1 & 3 & 2 & $1460+/-30 \mathrm{BP}$ & $660-590$ & $1360-1290$ & $\begin{array}{c}\text { BETA } \\
403222\end{array}$ \\
\hline
\end{tabular}

TABELA 1

Datações do sítio arqueológico Sambaqui Lagoa dos Freitas. Adaptado de Santos et al. (2018).

tingas herbáceas, arbustivas e arbóreas denominadas de Mata de Restinga (IBGE, 2012). Estas formações são de extrema importância para a fauna endêmica e migratória, pois servem como nichos de forrageio e nidificação (MMA-Brasil, 1999). A Mata Atlântica na região do Sambaqui Lagoa dos Freitas apresenta uma formação de Floresta Ombrófila Densa com árvores com até $20 \mathrm{~m}$ de altura (IBGE, 2012) que forma zonas ecotonais com a Mata de Restinga.

Dentro das áreas de Mata de Restinga se encontram as lagoas costeiras resultantes dos processos de transgressão e regressão marinha do Holoceno, entre 8000 e 2205 anos AP, processos que provocaram mudanças na paisagem local com a substituição dos grandes estuários e consolidação das formações arbóreas (Val-Peón et al., 2017).

O Sambaqui Lagoa dos Freitas está dentro de um contexto ambiental de estuário. Os ambientes estuarinos são zonas de transição entre o oceano e o continente que apresentam grande produtividade biológica e forte diversidade faunística (Miranda $e t$ al., 2002).

\section{METODOLOGIA}

A fauna foi inicialmente separada dos outros materiais arqueológicos e triada por grandes grupos taxonômicos. Em relação à identificação do material osteológico foram utilizadas as coleções de referência do Laboratório de Arqueologia Pedro Ignácio Schmitz (LAPIS) da Universidade do Extremo Sul Catarinense (UNESC). A nomenclatura científica da ictiofauna seguiu o sistema integrado de informação taxonômica (ITIS) e para a masto- fauna a lista anotada de mamíferos do Brasil (Paglia et al., 2012). Os dados sobre o comportamento e ecologia das espécies seguiu, para os peixes, Andrade Tubino et al. (2008) e, para os mamíferos, Paglia et al., (2012). Em relação à sua presença em ambientes estuarinos, os peixes foram divididos entre diádromos, estuarino-dependentes e oportunistas. Os mamíferos foram classificados em terrestres, semi-aquáticos e fossoriais.

No que se refere a recuperação e tratamento dos dados qualitativos e quantitativos, foram utilizados os índices comumente aplicados em trabalhos de zooarqueologia, como o NR (número de restos) e o NMI (número mínimo de indivíduos) (Chaix \& Méniel, 2005; Reitz \& Wing, 2008), seguindo os dois horizontes cronológicos delimitados pelas datações e a estratigrafia. Para a camada I, mais recente, o conjunto analisado compreende 4005 restos de vertebrados. O conjunto da camada II, mais antiga, tem um total de 10299 . Ao total foram analisados 14304 restos de vertebrados. Os restos de invertebrados não entraram no escopo desta análise por serem entendidos como elemento construtivo dos sambaquis (Klokler et al., 2010) mas uma análise rápida, realizada pelos autores deste estudo, identificou duas espécies de bivalves como predominantes nas amostras Mesodesma mactroides e Donax hanleyanus (Santos et al., 2018).

\section{Camada I}

Para os 4005 restos analisados nesta camada, o NMI total foi de 18 indivíduos com a identificação de peixes teleósteos, aves e mamíferos (Tabela 2). 


\begin{tabular}{|c|c|c|c|c|c|c|}
\hline \multirow{2}{*}{ Espécies } & \multicolumn{2}{|c|}{ Camada I } & \multicolumn{2}{|c|}{ Camada II } & \multirow{2}{*}{$\begin{array}{r}\text { Total } \\
\text { de NR } \\
\end{array}$} & \multirow{2}{*}{$\begin{array}{c}\text { Total } \\
\text { de NMI }\end{array}$} \\
\hline & NR & NMI & NR & NMI & & \\
\hline Teleostei & 329 & 13 & 1697 & 49 & 2026 & 62 \\
\hline Teleostei ind. & 280 & 0 & 1552 & 0 & 1832 & 0 \\
\hline Siluriformes & 44 & 9 & 112 & 25 & 156 & 34 \\
\hline Siluriformes ind. & 28 & 0 & 53 & 0 & 81 & 0 \\
\hline Ariidae & 16 & 9 & 59 & 25 & 75 & 34 \\
\hline Ariidae ind. & 3 & 2 & 14 & 1 & 17 & 3 \\
\hline Genidens sp. & 13 & 7 & 45 & 24 & 58 & 31 \\
\hline Perciformes & 5 & 4 & 33 & 24 & 38 & 28 \\
\hline Perciformes ind. & & & 2 & 0 & 2 & 0 \\
\hline Centropomidae & & & 1 & 1 & 1 & 1 \\
\hline $\begin{array}{l}\text { Centropomus cf. } \\
\text { paralellus }\end{array}$ & & & 1 & 1 & 1 & 1 \\
\hline Pomatomidae & & & 1 & 1 & 1 & 1 \\
\hline Pomatomus saltatrix & & & 1 & 1 & 1 & 1 \\
\hline Sciaenidae & 5 & 4 & 33 & 24 & 38 & 28 \\
\hline Sciaenidae ind. & & & 1 & 1 & 1 & 1 \\
\hline $\begin{array}{l}\text { Micropogonias } \\
\text { furnieri }\end{array}$ & 5 & 4 & 25 & 18 & 30 & 22 \\
\hline Menticirrhus littoralis & & & 2 & 2 & 2 & 2 \\
\hline Pogonias cromis & & & 1 & 1 & 1 & 1 \\
\hline Aves & 1 & 1 & 1 & 1 & 2 & 2 \\
\hline Aves ind. & 1 & 1 & 1 & 1 & 2 & 2 \\
\hline Mammalia & 113 & 4 & 138 & 7 & 251 & 11 \\
\hline Mammalia ind. & 87 & 0 & 93 & 0 & 180 & 0 \\
\hline Rodentia & 2 & 1 & 32 & 4 & 34 & 5 \\
\hline Rodentia ind. & 2 & 1 & 15 & 0 & 17 & 1 \\
\hline Cricetidae & & & 4 & 1 & 4 & 1 \\
\hline Cricetidae ind. & & & 4 & 1 & 4 & 1 \\
\hline Caviidae & & & 1 & 1 & 1 & 1 \\
\hline $\begin{array}{l}\text { Hydrochaerus } \\
\text { hydrochoeris }\end{array}$ & & & 1 & 1 & 1 & 1 \\
\hline Ctenomyidae & & & 12 & 2 & 12 & 2 \\
\hline Ctenomys minutus & & & 12 & 2 & 12 & 2 \\
\hline Perissodactyla & 1 & 1 & & & 1 & 1 \\
\hline Tapiriidae & 1 & 1 & & & 1 & 1 \\
\hline Tapirus terrestris & 1 & 1 & & & 1 & 1 \\
\hline Artiodactyla & 23 & 2 & 13 & 3 & 36 & 5 \\
\hline Artiodactyla ind. & 6 & 0 & 2 & 0 & 8 & 0 \\
\hline Cervidae & & & 1 & 1 & 1 & 1 \\
\hline Mazama sp. & & & 1 & 1 & 1 & 1 \\
\hline Tayassuidae & 17 & 2 & 10 & 2 & 27 & 4 \\
\hline Tayassuidae ind. & 17 & 2 & 10 & 2 & 27 & 4 \\
\hline Indeterminados & 3562 & 0 & 8463 & 0 & 12025 & $\mathbf{0}$ \\
\hline Total Geral & 4005 & 18 & 10299 & 57 & 14304 & 75 \\
\hline
\end{tabular}

TABELA 2

Fauna vertebrada identificada para o Sambaqui Lagoa dos Freitas para as camadas I e II. (ind.= indeterminado)

O material mostrou uma alta frequência de indeterminação com 3562 restos sem atribuição de classe, $89 \%$ do total amostrado da Camada I, fato explicado pela forte fragmentação dos restos. Os peixes teleósteos identificados somaram um NR de
329 restos e NMI de 13 indivíduos. Os mamíferos corresponderam a um NR de 113 restos e NMI de 4 indivíduos e as aves tiveram apenas um elemento reconhecido (NR e NMI=1). Para a Camada I, 280 restos atribuídos como pertencentes à classe Teleostei não tiveram possibilidade de identificação específica. Entre os restos de peixes teleósteos identificados, a família Ariidae é a de maior representatividade na amostra $(\mathrm{NR}=16$ e $\mathrm{NMI}=8)$, seguida da família Sciaenidae (NR=5 e NMI=4) (Tabela 3).

\begin{tabular}{l|cc|cc|c|c}
\hline \multirow{2}{*}{ Classe } & \multicolumn{2}{|c|}{$\begin{array}{c}\text { Camada I } \\
(\mathbf{4 9 5 - 3 0 5} \text { cal. AP) }\end{array}$} & \multicolumn{2}{|c|}{$\begin{array}{c}\text { Camada II } \\
(\mathbf{1 3 6 0 - 1 2 9 0} \text { cal. AP) }\end{array}$} & $\begin{array}{c}\text { Total } \\
\text { NR }\end{array}$ & $\begin{array}{c}\text { Total } \\
\text { NMI }\end{array}$ \\
\cline { 2 - 5 } Teleostei & NR & NMI & NR & NMI & & \\
Ave & 329 & 13 & 1697 & 49 & 2026 & 62 \\
Mammalia & 1 & 1 & 1 & 1 & 2 & 2 \\
Classe Ind. & 3562 & - & 8463 & - & 12025 & - \\
Total Geral & 4005 & 18 & 10299 & 57 & 14304 & 75 \\
\hline
\end{tabular}

TABELA 3

Número de Restos (NR). Número Mínimo de Indivíduos (NMI) por classes de vertebrados de cada camada.

Os restos identificados até somente a nível da família Ariidae foram indentificados através do basioccipital e do neurocrânio e a única espécie encontrada desta família o Genidens sp. foi identificado através dos otólitos (lapillus). Dos restos de Ariidae, um otólito e o basioccipital estavam com marcas de queima. Os Sciaenidae com a espécie $M$. furnieri foram identificadas com os otólitos sagitas. Entre os restos de mamíferos, 87 não puderam ser determinados. A família Tayassuidae representa os mamíferos mais abundantes nesta camada $(\mathrm{NR}=17$ e NMI=2), identificados por diversos dentes, um fragmento de mandíbula (Figura 2A) e uma epífise proximal de ulna. Destes apenas um dente molar se encontra com sinais de queima direta (Figura 2b). Um indivíduo de Tapirus terrestris foi identificado a partir de um único dente molar (NR e NMI=1) enquanto os roedores puderam ser determinados apenas a nível de classe $(\mathrm{NR}=2$ e $\mathrm{NMI}=1)$.

\section{Camada II}

Para a Camada II, o total foi de 10299 restos analisados e um NMI total de 57 indivíduos identificados de peixes ósseos, aves e mamíferos (Tabela 2). O material desta camada também mostrou uma alta frequência de indeterminação com 8463 restos sem atribuição, $82 \%$ do total da Camada II. Os peixes 

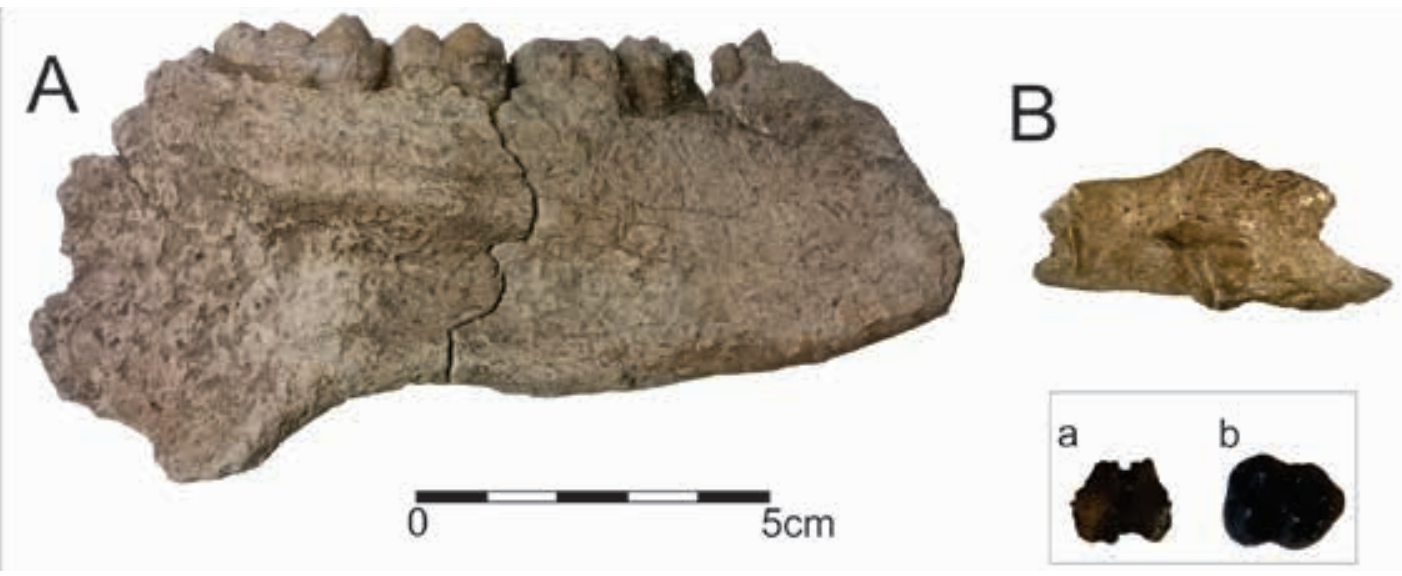

FIGURA 2

A) Fragmento de mandíbula esquerda de Tayassuidae; B) Fragmento proximal de ulna esquerda de Mazama sp.; a) basioccipital de Ariidae com termo-alteração; b) terceiro molar superior de Tayassuidae carbonizado.

teleósteos são majoritários na amostra $(\mathrm{NR}=1697$ e NMI $=49)$ em relação aos mamíferos $(\mathrm{NR}=138 \mathrm{e}$ $\mathrm{NMI}=7)$ e aves $(\mathrm{NR}=1 \mathrm{e} \mathrm{NMI}=1)$. A camada II tem uma maior diversidade no número de famílias e espécies identificadas tanto em relação aos teleósteos como aos mamíferos Entre os restos de mamíferos, puderam ser determinados os taiassuídeos, roedores e um cervídeo (Mazama sp.) (Figura 2B).

Em relação aos peixes, 1607 dos restos não puderam ser identificados com mais precisão. Entre os restos determinados, a família Ariidae foi a mais frequente na amostra $(\mathrm{NR}=59$ e $\mathrm{NMI}=25)$, seguida da família Sciaenidae (NR= 29 e NMI=22), Pomatomidae (NR e NMI=1) e Centropomidae (NR e NMI= 1) (Tabela 2).

As diferentes espécies da família Ariidae foram identificadas em maioria pelos otólitos, neurocrânio, basioccipital, supraoccipital e processo transcapular. Para as espécies da família Sciaenidae foram utilizados como elementos diagnósticos os otólitos e uma placa faríngea. As famílias e espécies de Centropomidae e Pomatomidae foram identificadas a partir de otólitos.

\section{Comparação entre as camadas}

O total de material analisado para as duas camadas soma um NR de 14304 restos e um NMI de 75 indivíduos (Tabela 2). A Camada I compreende o menor número de restos de fauna em comparação com a camada II, porém tem uma frequência de in- determinação levemente mais alta (89\% Camada I e $82 \%$ para a Camada II). Ambas as camadas têm esta alta frequência de indeterminação devido à grande fragmentação e a falta de partes anatômicas diagnósticas, como epífises de ossos longos, otólitos ou dentes (Tabela 3).

\section{Termo-alterações}

Dos restos com alteração antrópica na Camada I se sobressaem os ossos termo-alterados, com indícios de queima por ação direta do fogo. Os indeterminados somaram $60 \%$ dos restos com termo-alterações $(\mathrm{NR}=41)$, seguido dos Teleostei com 22\% $(\mathrm{NR}=15)$ e a classe Mammalia com $18 \%(\mathrm{NR}=12)$ (Figura 3). Para a Camada II, 68\% dos restos indeterminados $(\mathrm{NR}=77), 25 \%(\mathrm{NR}=29)$ dos restos de peixes ósseos e 7\% (NR=8) dos mamíferos se encontram termo-alterados (Figura 2 a e b; Figura 3 ).

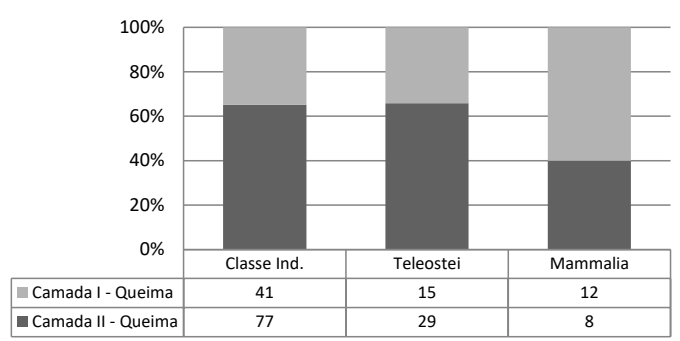

FIGURA 3

Restos ósseos com termo-alterações para as duas camadas do Sambaqui Lagoa dos Freitas. 
Ecologia das espécies capturadas

A partir dos espectros de fauna, observamos, entre os peixes, uma forte presença de espécies de ambientes estuarinos e costeiros enquanto entre os mamíferos, se observa a presença de espécies terrestres. A ecologia das espécies de peixes identificadas nas duas camadas (Tabela 4) mostra a presença de bagres do gênero Genidens, considerados estuarinos-dependente por ter seu ciclo de vida relacionado aos estuários, onde desovam e completa sua maturidade sexual, retornando ao mar após a idade adulta, completando seu ciclo vital (Oliveira \& Bemvenuti, 2006). Semelhante aos bagres, a corvina, M. furnieri, desova no mar, porém seus alevinos migram para os estuários onde chegam a idade adulta, posteriormente retornando ao mar (Oliveira \& Bemvenuti, 2006). Espécies como a anchova (P. saltatrix), o papa-terra (M. littoralis) e a miraguaia ( $P$. cromis) são oportunistas que utilizam os estuários para proteção e forrageio (Andrade Tubino et al., 2008; Fischer et al., 2011). Outra espécie que suporta grandes salinidades é o robalo (C. paralellus), uma espécie diádroma, que pode efetuar constantes movimentações entre o mar e o estuário (Andrade Tubino et al., 2008).

\begin{tabular}{llll}
\hline \multirow{2}{*}{ Espécies } & \multicolumn{2}{c}{ Locomoção } \\
\cline { 2 - 4 } & ED & EO $\quad$ D \\
\hline Genidens sp. & & & \\
Centropomus cf. paralellus & & & \\
Pomatomus saltatrix & & & \\
Menticirrhus littoralis & & & \\
Micropogonias furnieri & & & \\
Pogonias cromis & & & \\
\hline
\end{tabular}

TABELA 4

Ecologia das espécies de peixes identificadas: (ED) Estuarino-dependente; (EO) Estuarino-oportunista; (D) Diádroma.

Todas as espécies identificadas de mamíferos possuem ecologia de hábitos terrestres. Os taiassuídeos, animais de porte médio a grande, podem ser de duas espécies que eventualmente ocorriam na área de estudo, o Tayassu pecari e Pecari tajacu (Bôlla et al., 2017). Indivíduos destas espécies apresentam hábitos sociais gregários e estão em constante forrageio entre as floretas e os campos litorâneos (Reis et al., 2006). Porém, as duas espécies se diferenciam em alguns comportamentos, como por exemplo, os P. tajacu são animais sedentários ficando em locais próximos ao seu nascimento enquanto o $T$. pecari percorre longas distâncias (García et al., 2009). Já a anta, T. terrestris, possui hábito de deslocação noturno e está sempre associada às áreas com vegetação arbórea com cursos d'água perenes (Medici et al., 2012). Assim também como os veados do gênero Maza$m a$, que preferem ambientes florestados, mas que podem ocorrer em áreas de ecótonos da planície costeira (Reis et al., 2006). Outro mamífero identificado no material analisado foi a capivara, $H$. hydrochoeris, que possui hábito gregário, porte médio e está associada aos cursos d'água, principalmente na planície costeira onde ocorre uma maior incidência de recursos dulcícolas (Reis et al., 2006). Outros roedores foram encontrados na amostra, o tuco-tuco, C. minutus, que possui hábitos fossoriais e vive em galerias subterrâneas, e roedores de pequeno porte da família Cricetidae. A ocorrência destes pequenos roedores no sítio não parece ser resultado de atividade antrópica por conta do tamanho reduzido e dificuldade de captura em relação ao baixo retorno em carne. A presença destes animais poderia estar ligada a questões tafonômicas associadas a morte natural no local ou a predação de outros animais, como a coruja buraqueira (Athene cunicularia), que regurgita partes não digeridas como ossos e pelos em forma de pelotas (egagrópilos) em topos de dunas (Zílio, 2005).

\section{DISCUSSÃO}

A análise dos conjuntos zooarqueológicos das duas camadas do sambaqui Lagoa dos Freitas mostra a exploração de uma grande diversidade de espécies vertebradas (12 espécies), apesar da alta frequência de restos indeterminados. Embora existam diferenças entre as camadas I e II, a começar pelo tamanho das amostras, elas indicam as mesmas tendências gerais e, por esta razão, serão discutidas aqui em conjunto antes de uma comparação final.

\section{Pesca}

A frequência de NMI da fauna vertebrada do sambaqui Lagoa dos Freitas indica predominância de dois peixes ósseos, o bagre Genidens sp. e a cor- 
vina $M$. furnieri. Estes animais, por dependerem dos estuários e viverem em grandes cardumes, poderiam potencialmente ser recursos disponíveis o ano inteiro e em grandes quantidades na Lagoa dos Freitas, oportunizando assim sua pesca preferencial.

Para pensarmos em exemplos de pesca destes animais em sambaquis, nos sambaquis do complexo lagunar de Saquarema no Rio de Janeiro foram encontradas espinhas de peixes modificadas associadas a pesca da corvina e pontas em ossos polidos que estariam relacionados à captura de bagres da família Ariidae (Guimarães, 2013).

A partir de observações da pesca tradicional em comunidades caiçaras, Figuti (1998) observou as armadilhas utilizadas na pesca destas espécies e os seus rendimentos específicos associados algumas técnicas como potencialmente praticadas pelos grupos sambaquieiros da Baixada Santista. O bagre seria capturado com armadilhas do tipo covo, constituída por uma estrutura em bambu de pequeno porte colocada ao fundo da água e amarrada em uma árvore, sendo visitada uma ou mais vezes ao dia para a retirada do pescado aí capturado. Para a corvina, é citada a armadilha de rede de laço, constituída por uma grande e larga rede manejada por pessoas que cercam o cardume enquanto outra pessoa afugenta o cardume em direção ao fundo da rede.

A pesca de espécies lagunares e marinhas por grupos costeiros foi também registrada no litoral norte do Rio Grande do Sul, em três diferentes sítios arqueológicos, Itapeva (3.130 \pm 40 anos AP), Recreio (3.350 \pm 50 AP) e Dorva $(1.110 \pm 40$ anos AP) (Wagner, 2009). Nos dois sítios mais antigos, mais próximos do mar, predominou a exploração de espécies marinhas como as tainhas (Mugil sp.), peixes da família Sciaenidae (M. furnieri, Menticirrhus sp. e P. Cromis) e bagres marinhos (Genidens sp.) Já o sítio Dorva, um sambaqui com datas próximas à ocupação inicial do Sambaqui Lagoa dos Freitas, os peixes mais capturados são pertencentes a família Cichlidae, peixes dulcícolas, o que foi relacionado com a proximidade do sítio com a Lagoa dos Quadros (Hilbert, 2011). Ricken et al. (2016) estudando o sambaqui tardio Praia do Paraíso em Arroio do Sal, também litoral norte do Rio Grande do Sul, identificou espécies estuarinas-marinhas como Genidens sp. e M. furnieri.

A partir dos exemplos citados, a pesca no Sambaqui Lagoa dos Freitas se mostra dentro do mesmo escopo de exploração de corpos de água estuarinos-marinhos de sítios tardios de regiões próximas ao AERUM e igualmente, em uma perspectiva de longa duração, englobada no domínio das práticas de pesca associadas aos grupos sambaquieiros.

\section{Caça}

No Sambaqui Lagoa dos Freitas foram identificados diversos mamíferos de médio e grande porte (Taiassuidae, T. terrestris, H. hidrochaeris e Mazama sp.). A caça de mamíferos sempre fez parte das práticas dos grupos mesmo com a predileção destes por espécies marinhas e semi-aquáticas (Castilho \& Simões-Lopes, 2001, 2005, 2008; Castilho, 2005).

Borges (2015), a partir do estudo dos sambaquis Piaçaguera, Mar Casado, Maratuá e Buracão na Baixada Santista, identificou restos de anta $(T$. terrestris), porco do mato (Tayassuidae) e capivara (H. hydrochaeris) com marcas antrópicas ligadas ao tratamento da carcaça (marcas de corte, descarne e desarticulação além de termo-alterações).

No sítio sambaqui Jabuticabeira II em Jaguaruna, litoral sul de Santa Catarina, restos de mamíferos foram encontrados junto a sepultamentos humanos e a ausência de marcas nesses restos levou a hipótese de terem sido utilizados como oferendas nos rituais funerários (Klokler, 2012).

Os trabalhos de Castilho \& Simões-Lopes (2005) identificaram 37 fragmentos de capivara apresentando registros de modificações antrópicas no sítio arqueológico Porto do Rio Vermelho II, localizado na Ilha de Santa Catarina e datado entre 1735 a 1067 AP., mostrando como esses animais contribuíam significativamente na economia dos grupos litorâneos.

Para o sítio Lagoa de Freitas a presença destes animais terrestres demonstra mais uma vez que, embora as populações litorâneas estejam intimamente ligadas aos recursos costeiros, os ambientes interioranos com áreas de vegetação densa eram também frequentemente explorados.

\section{Tratamento da fauna}

A análise das termo-alterações não mostrou diferenças no tratamento da fauna nos diferentes 
períodos de ocupação do sítio (Figura 3). Segundo trabalhos experimentais de Villagrán (2008) e Cardoso (2018), as termo-alterações observadas macroscopicamente nos vestígios seriam o resultado da exposição direta dos ossos a altas temperaturas. No caso do Lagoa de Freitas elas também parecem indicar a prática de processamento dos animais pela ação direta do fogo. Além destas termo-alterações, não foram observadas outras marcas antrópicas, como marcas de corte nos ossos, associadas a desarticulação e descarne das carcaças.

\section{Exploração de ambientes}

O Sambaqui Lagoa dos Freitas está em uma área ambientalmente heterogênea e com alta produtividade biológica com a existência de ambientes diversificados no entorno do sítio (Figura 4) (Val-Péon et al., 2017). A baixa incidência de mamíferos em comparação aos peixes nas amostras zooarqueológicas mostra uma preferência pela exploração dos ambientes aquáticos em ambos os períodos de ocupação, embora a caça se mostre uma atividade frequente.

Os mamíferos terrestres eram possivelmente caçados nos ambientes de vegetação arbórea quando estivessem circulando entre o interior e o litoral. Estes ambientes também poderiam ser fonte de recursos em diversas espécies de aves, répteis e anfíbios e frutos disponíveis em certas épocas do ano que serviriam como atrativos para a grande fauna herbívora, como veados, porcos do mato e antas.

Os ambientes estuarinos e lagunares forneciam a maior parte dos recursos a partir da pesca de peixes residentes e oportunistas (Ariidae, Sciaenidae, Pomatomidae e Centropomidae), e como espaço de captura de fauna semiaquática, como as capivaras. Assim, a área da lagoa era possivelmente a mais frequentada e explorada pelos grupos do Lagoa de Freitas. Além da circulação no espaço aquático, os ambientes de restinga próximos a lagos estariam associados a recursos faunísticos de espécies migratórias e residentes, área de nidificação e forrageio em determinadas épocas sazonais. Ao final, estes mesmos ambientes poderiam também ser área de captura de recursos de animais invertebrados, como bancos de moluscos de $D$. hanleyanus e M. mactroides, também espécies identificadas no sítio.

\section{CONCLUSÃO}

A partir da análise zooarqueológica podemos observar a continuidade nas formas de apreensão de recuperação de recursos entre os dois horizontes cronológicos do sítio. A pequena diferença no espectro de fauna, principalmente em termos quantitativos, parece estar relacionada a estratigrafia, pois a Camada II, com maior número de restos de vertebrados, continha também maior quantidade de conchas em comparação com a Camada I, o que poderia ter proporcionado um ambiente químico com melhores possiblidades de preservação para os restos faunísticos (Villágran et al., 2009).

No que se refere a estratégias de captura e exploração de ambientes, podemos perceber que os grupos humanos do Sambaqui Lagoa dos Freitas tinham como foco principal os peixes estuarinos-dependentes (Ariidae e Scienidae) explorados nos ambientes aquáticos do entorno do sítio, sobretudo a lagoa. Estes resultados mostram uma exploração mais intensa de ambientes estuarinos em compara-

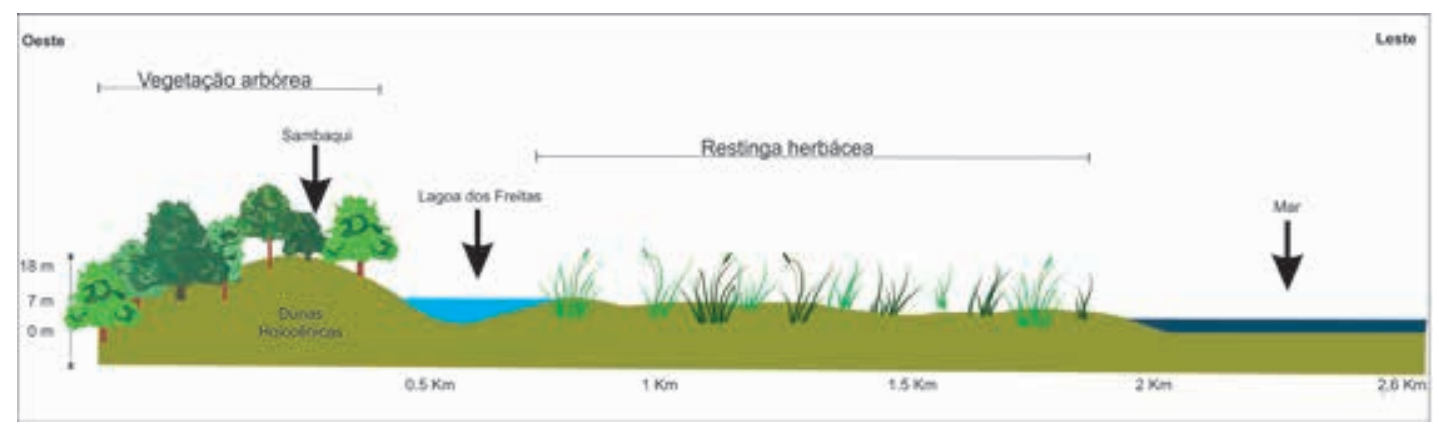

FIGURA 4

Área de caça, pesca e coleta nas adjacências do Sambaqui Lagoa dos Freitas. 
ção aos ambientes terrestres e o desenvolvimento de atividades de captura associadas aos ecossistemas aquáticos, no caso, principalmente, a pesca. As espécies identificadas podem ter sido pescadas nas lagoas, tanto as estuarinas residentes como oportunistas. Além disso, os recursos oriundos da pesca podem ser potencialmente explorados o ano inteiro em grande quantidade. Embora com a proeminência da pesca, os ambientes terrestres florestados também eram explorados constantemente, o que nos mostra a co-existência de diferentes estratégias de captura e exploração de ambientes.

No que se refere ao modo de apreensão de recursos e usos dos espaços lagunares não se observou no sambaqui Lagoa dos Freitas; em geral, diferenças com períodos anteriores de ocupações litorâneas no sul do Brasil ou em outras regiões com a predominância das atividades de pesca e exploração de recursos marino-lagunares. Neste sentido, as análises zooarqueológicas mostram muito mais continuidade que diferença com períodos mais recentes, porém, no caso do sambaqui Lagoa de Freitas, a pesquisa precisa ser ampliada para um melhor entendimento de possíveis diferenças entre os horizontes mais antigo e o mais recente, talvez associado a chegada de populações ceramistas na região (Santos et al., 2018). Assim, este trabalho também se insere no contexto de pesquisas buscando o entendimento dos sambaquis tardios e as novas formas de uso e vida nos ambientes litorâneos no sul do Brasil quando da chegada de novas populações portadoras de tecnologias e modos de vida diferenciados (Iriarte et al., 2017).

\section{REFERENCIAS}

Andrade Tubino, M.F. De; Ribeiro, A.L.R. \& Vianna, M. 2008: Organização espaço-temporal das ictiocenoses demersais nos ecossistemas estuarinos brasileiros: uma síntese. Oecologia Australis 12: 640-661.

Bôlla, D.A.S.; Ceron, K.; Carvalho, F.; Mattia, D.L. De; Luiz, M.R.; Panatta, K.A.; Pavei, D.D.; Mendonça, R.Á. \& Zocche, J.J. 2017: Mastofauna terrestre do sul de Santa Catarina: mamíferos de médio e grande porte e voadores. Revista Tecnologia e Ambiente 23: 61-78.

Borges, C. 2015: Analyse archéozoologique de l'exploitation des animaux par les populations de pêcheurschasseurs-cueilleurs des sambaquis de Baixada Santista, Brésil, entre 5000 et 2000 BP. Tese de doutorado, Muséum National d'Histoire Naturelle, Paris.
Cardoso, J.M. 2018: O sítio costeiro Galheta IV: uma perspectiva zooarqueológica. Dissertação de mestrado, Museu de Arqueologia e Etnologia. Universidade de São Paulo, São Paulo.

Cardoso, J.M.; Júnior, J.A.M.; Farias, D.S.E. De \& DeBLASIS, P. 2014: Zooarqueologia do sítio Galheta IV: um enfoque nos vestígios do pinguin-de-magalhães (Spheniscus magellanicus, Spheniscidae). In: Zocche, J.J.; Campos, J.B.; Almeida, N. \& Ricken, C. (orgs.): Arqueofauna e Paisagem: 155-169. Erechim.

Castilho, P.V. de 2005: Mamíferos marinhos: um recurso de populações humanas pré-coloniais do litoral catarinense. Tese de doutorado, Universidade Federal do Paraná, Curitiba.

Castilho, P.V. De \& Simões-Lopes, P.C. 2001: Zooarqueologia de mamíferos aquáticos e semi-aquáticos da Ilha de Santa Catarina, sul do Brasil. Revista Brasileira de Zoologia 18(3): 719-727.

- 2005: A capivara, Hydrochoerus hydrochaeris (Mammalia, Rodentia), no sítio arqueológico SC-PRV-02, Ilha de Santa Catarina, Santa Catarina. Biotemas 18: 203-218.

- 2008: Sea mammals in archaeological sites on the southern coast of Brazil. Revista do Museu de Arqueologia e Etnologia 18: 101-113.

Chaix, L. \& Méniel, P. 2005: Manual de Arqueozoología. Editorial Ariel, Barcelona.

Deblasis, P.; Fish, S.K.; Gaspar, M.D. \& Fish, P.R. 1998: Some references for the discussion of complexity among sambaqui moundbuilders from the Southern shores of Brazil. Revista de Arqueologia Americana 15: 75-105.

Deblasis, P.; Kneip, A.; Scheel-Ybert, R.; Giannini, P.C. \& Gaspar, M.D. 2007: Sambaquis e Paisagem: dinâmica natural e arqueologia regional no litoral do sul do Brasil. Arqueología Suramericana 3: 29-61.

Deblasis, P. \& Gaspar, M. 2009: Os sambaquis do sul catarinense: retrospectiva e perspectiva de dez anos de pesquisas. Especiaria: Cadernos de Ciências Humanas 11/12: 83-125.

FIGUTI, L. 1998: Estórias de arqueo-pescador: considerações sobre a pesca nos sítios de grupos pescadores-coletores do litoral. Revista de Arqueologia da SAB 11: $57-70$.

Fischer, L.G.; Pereira, L.E.D. \& VieIRA, J.P. 2011: Peixes estuarinos e costeiros. Gráfica Pallotti, Rio Grande.

Garcia, A.R.; Kahwage, P.R. \& Ohashi, O.M. 2009: Aspectos reprodutivos de caititus (Tayassu tajacu). Revista brasileira de reprodução animal 33(2): 71-81.

Giannini, P.C.F.; Villagrán, X.S.; Fornari, M.; Rodrigues, D.; Menezes, P.; Tanaka, A.P.; Assunçao, D.; 
Deblasis, P. \& Amaral, P. 2010: Interações entre evolução sedimentar e ocupação humana pré-histórica na costa centro-sul de Santa Catarina, Brasil. Boletim do Museu Paraense Emílio Goeldi, Série Ciências Humanas 5: 105-128.

GUIMARÃES, M.B. 2013: Fishing strategies among prehistoric populations at Saquarema Lagoonal Complex, Rio de Janeiro, Brazil. Anais da Academia Brasileira de Ciências 85: 415-429.

HiLbert, L.M. 2011: Análise ictioarqueológica dos sítios: Sambaqui do Recreio, Itapeva e Dorva, municípios de Torres e Três cachoeiras, Rio Grande do Sul, Brasil. Dissertação de Mestrado, Pontifícia Universidade Católica do Rio Grande do Sul, Porto Alegre.

IBGE - Instituto Brasileiro de Geografia e Estatística 2012: Manual Técnico da Vegetação Brasileira: $2^{a}$ edição revista e ampliada. Sistema Fitogeográfico. Inventário das Formações Florestais e Campestres. Técnicas e Manejo de Coleções Botânicas. Procedimentos para Mapeamentos. IBGE, Brasília.

Iriarte, J.; Deblasis, P.; De Souza, J.G. \& Corteletti, R. 2017: Emergent complexity, changing landscapes, and spheres of interaction in southeastern South America during the middle and late Holocene. Journal of Archaeological Research 25(3): 251-313.

ITIS - Integrated Taxonomic Information System on-line database 2018. http://www.itis.gov.

KLOKLER, D.M. 2012: Consumo ritual, consumo no ritual: festins funerários e sambaquis. Habitus 10: 83-104.

Klokler, D.; Villagrán, X.S.; Giannini, P.C.F.; Peixoto, S. \& Deblasis, P. 2010: Juntos na costa: zooarqueologia e geoarqueologia de sambaquis do litoral sul catarinense. Revista do Museu de Arqueologia e Etnologia 20: 53-75.

Medici, E.P.; Flesher, K.; Beisiegel, B. De M.; Keuroghlian, A.; Desbiez, A.L.J.; Gatti, A.; Pontes, A.R.M.; Figueiredo, C.B. De; Tófoli, C.F. De; Junior, E.A.M.; Azevedo, F.C. De; Pinho, G.M. DE; JúNIOR, T. Da S.S.; Morais, A.A. De; Mangini, P.R. \& ALMEIDA, L.B. De 2012: Avaliação do Risco de Extinção da anta brasileira: Tapirus terrestris (Linnaeus, 1758), no Brasil. Biodiversidade Brasileira 3: 103-116.

Miranda, L.B. De; Castro, B.M. De \& Kuerfye, B. 2002: Princípios de Oceanografia Física de Estuários. EDUSP, Acadêmica 42, São Paulo.

MMA - Brasil - Ministério do Meio Ambiente 1999: Resolução CONAMA $n^{\circ} 261$, de 30 de junho de 1999. http://www2.mma.gov.br/port/conama/legiabre. cfm?codlegi=260, Brasília.

NishidA, P. 2007: A coisa ficou preta: estudo do processo de formação da terra preta do sítio arqueológico Jabuticabeira II. Tese de doutorado, Museu de Ar- queologia e Etnologia - Universidade de São Paulo, São Paulo.

Oliveira, A.F. De \& Bemvenuti, M.D.A. 2006: O ciclo de vida de alguns peixes do estuário da Lagoa dos Patos, RS: informações para o Ensino Fundamental e Médio. Caderno Ecológico Aquático 1: 16-29.

Paglia, A.P.; Fonseca, G.A.B. Da; Rylands, A.B.; HerRmann, G.; Aguiar, L.M.S.; Chiarello, A.G.; Leite, Y.L.R.; Costa, L.P.; Siciliano, S.; KIERULFF, M.C.M.; Mendes, S.L.; Mittermeier, R.A. \& Patton, J.L. 2012: Lista Anotada dos Mamíferos do Brasil. Occasional Papers in Conservation Biology 6: 1-76.

Reis, N.; Peracchi, A.; Pedro, W. \& Lima, I. 2006: Mamíferos do Brasil. Londrina.

ReITZ, E.J. \& WING, E.S. 2008: Zooarchaeology. Cambridge University Press, Cambridge.

Ricken, C.; Heberts, A.L.; Wagner, G.P. \& MalabarBA, L.R. 2016: Coastal hunter-gatherers fishing from the site RS-AS-01, Arroio do Sal, Rio Grande do Sul, Brazil. Pesquisas, Antropologia 72: 209-224.

RogGe, J.H. \& Arnt, F. 2006: O Sambaqui de Içara SC-IÇ-06. Pesquisas, Antropologia 63: 13-16.

Rosa, A.O. 1999: Remanescentes de fauna e flora. Pesquisas, Antropologia 55: 31-64.

- 2006: Composição e diversidade da arqueofauna dos sítios de Içara: SC-IÇ-01 e SC-IÇ-06. Pesquisas, Antropologia 63: 33-54.

Santos, M.C.P.; Pavei, D.D. \& Campos, J.B. 2018: Sambaqui Lagoa dos Freitas, Santa Catarina: estratigrafia, antiguidade, arqueofauna, e cultura material. Revista Memorare 5(1): 157-196.

Schmiтz, P.I. 1999: Içara: um jazigo mortuário no litoral de Santa Catarina. Pesquisas, Antropologia 55.

Tamiozzo, V.; Schmitz, P.I. \& Rosa, A.O. 2008: Investigações zooarqueológicas no sambaqui SC-IÇ-06, Içara - SC. Canindé - Revista do Museu de Arqueologia de Xingó 12: 105-130.

TeixeIra, D.R. 2006: Arqueofauna do sítio SC-IÇ-06. Pesquisas, Antropologia 63: 17-31.

Val-Peón, C.; Cancelli, R.R.; Santos, L. \& Soares, A.L. 2017: Prehistoric occupation and palaeoenvironmental changes along Santa Catarina's Coastal Plain, Brazil: an integrated approach based on palynological data. Journal of Archaeological Science: Reports. doi:10.1016/j.jasrep.2017.11.017.

VILLAGRÁn, X.S. 2008: Análise de arqueofácies na camada preta do sambaqui Jabuticabeira II. Dissertação de mestrado, Museu de Arqueologia e Etnologia-Universidade de São Paulo, São Paulo.

Villagrán, X.S; Giannini, P.C.F. \& Deblasis, P. 2009: Archaeofacies analysis: using depositional attributes 
to identify anthropic processes of deposition in a monumental shell mound of Santa Catarina State, southern Brazil. Geoarchaeology 24: 311-335.

W AGNER, G.P. 2009: Sambaquis da barreira da Itapeva: uma perspectiva geoarqueológica. Tese de doutorado, Pontifícia Universidade Católica do Rio Grande do Sul, Porto Alegre.
Zílio, F. 2005: Estudo do nicho ecológico de duas aves de rapina (Falco sparverius $e$ Athene cunicularia) em uma região de dunas do Rio Grande do Sul, Brasil. Dissertação de mestrado, Instituto de Biociências, Universidade Estadual Paulista, Rio Claro. 